\title{
A Prospective Observational Study of Osimertinib for Chemo-Naive Elderly Patients with EGFR Mutation-Positive Non-Small Cell Lung Cancer
}

\author{
Satoshi Igawa' \\ Masashi Kasajima' \\ Taihei Ono' \\ Takahiro Ozawa' \\ Mikiko Kakegawa' \\ Seiichiro Kusuhara' \\ Takashi Sato (D) \\ Yoshiro Nakahara' \\ Tomoya Fukui ${ }^{1}$ \\ Masanori Yokoba ${ }^{2}$ \\ Masaru Kubota $\mathbb{D}^{2}$ \\ Hisashi Mitsufuji ${ }^{3}$ \\ jiichiro Sasaki ${ }^{4}$ \\ Katsuhiko Naoki ${ }^{1}$ \\ 'Department of Respiratory Medicine, \\ Kitasato University School of Medicine, \\ Sagamihara-city, Kanagawa, Japan; ${ }^{2}$ School of \\ Allied Health Sciences, Kitasato University, \\ Sagamihara-city, Kanagawa, Japan; ${ }^{3}$ Kitasato \\ University School of Nursing, Sagamihara- \\ city, Kanagawa, Japan; ${ }^{4}$ Kitasato University \\ School of Medicine, Research and \\ Development Center for New Medical \\ Frontiers, Kitasato University School of \\ Medicine, Sagamihara-city, Kanagawa, Japan
}

Background: The clinical outcomes of elderly patients with EGFR-mutated non-small cell lung cancer (NSCLC) who are treated with osimertinib have not been sufficiently evaluated. This study aimed to assess the efficacy and safety of osimertinib in elderly chemotherapynaive patients with NSCLC harboring sensitive EGFR mutations.

Patients and Methods: We assessed the clinical effects of osimertinib as a first-line treatment for elderly NSCLC patients ( $\geq 75$ years of age) with an exon 19 deletion or exon $21 \mathrm{~L} 858 \mathrm{R}$ mutation in EGFR. All patients were administered $80 \mathrm{mg} /$ day osimertinib as initial treatment.

Results: Forty-three patients ( 24 women and 19 men) with adenocarcinoma who were treated between August 2018 and July 2021 were included in this study; their median age was 79 years (range, 75-90 years). The overall objective response rate was $60.5 \%$. The median progression-free survival (PFS) and time to treatment failure (TTF) of the entire patient population were 22.1 months and 14.6 months, respectively. The most common adverse event was rash acneiform (42\%), followed by diarrhea $(33 \%)$ and paronychia $(28 \%)$; none of these were grades $\geq 3$. Interstitial lung disease developed in 8 patients $(18.6 \%)$; however, no treatment-related deaths occurred. Multivariate analysis identified performance status and disease stage as predictors of PFS and TTF.

Conclusion: Considering the findings of this study and despite an observed discordance between PFS and TTF, osimertinib appears to be an effective and safe treatment option in elderly patients with advanced NSCLC harboring sensitive EGFR mutations. To obtain conclusive results, further studies in a larger elderly population are warranted.

Keywords: non-small cell lung carcinoma, chemotherapy-naïve patients, efficacy

\section{Introduction}

Lung cancer is a major cause of cancer-related deaths, and non-small cell lung cancer (NSCLC) accounts for approximately $85 \%$ of all such malignancies. ${ }^{1}$ In recent years, epidermal growth factor receptor (EGFR) tyrosine kinase inhibitors (TKIs) have markedly improved the prognoses of patients with NSCLC harboring EGFR-activating mutations. Based on positive results from Phase III trials, ${ }^{1-8}$ EGFR-TKIs have become standard regimens for such patients, and several EGFRTKIs are currently approved as first-line treatments for EGFR mutation-positive NSCLC in Japan. These include the first-generation TKIs erlotinib and gefitinib, second-generation TKIs afatinib and dacomitinib, and third-generation TKI osimertinib. Osimertinib is a mono-anilino-pyrimidine compound that
Correspondence: Satoshi lgawa Department of Respiratory Medicine, Kitasato University School of Medicine, I-I5-I, Kitasato, Minami-ku, Sagamiharacity, Kanagawa, 252-0374, Japan

Tel +8I-42-778-8506

$\mathrm{Fax}+8 \mathrm{I}-42-778-6412$

Email igawa@kitasato-u.ac.jp 
irreversibly and selectively targets EGFR-TKI-sensitizing and T790M-resistant forms of mutated EGFR while sparing the wild-type gene.

Two-thirds of all new lung cancer diagnoses are in patients aged $>65$ years, with this rate anticipated to increase owing to the growing proportion of the elderly population. ${ }^{9,10}$ Accordingly, greater attention is being paid to improving treatment strategies for elderly patients with lung cancer. It has been reported that such patients showed very high response rates to first-line gefitinib and erlotinib, with prolonged survival times achieved. ${ }^{11-18}$ Additionally, Japanese Phase II studies have indicated the efficacy and safety of first-line afatinib in elderly EGFR mutationpositive patients with NSCLC. ${ }^{19,20}$ The FLAURA trial included patients of all ages and demonstrated that the safety profile of osimertinib was similar to that of gefitinib and erlotinib, ${ }^{8}$ indicating that osimertinib may be a feasible intervention for elderly patients. However, existing data remain insufficient to determine the efficacy of osimertinib in elderly patients with NSCLC who harbor $E G F R$-activating mutations.

Hence, the aim of this prospective observational study was to evaluate the efficacy and safety of first-line osimertinib for patients with advanced NSCLC harboring sensitive EGFR mutations.

\section{Patients and Methods}

\section{Patient Selection}

We conducted a prospective observational cohort study at Kitasato University Hospital between August 2018 and July 2021 to evaluate the efficacy and safety of osimertinib in patients with EGFR mutation-positive advanced NSCLC who were 75 years of age or older. The eligibility criteria for this study were as follows: histologically or cytologically confirmed NSCLC harboring either an exon 19 deletion or exon 21 L858R mutation in EGFR, stage IIIB-IV disease with postoperative recurrence according to the new Union for International Cancer Control criteria (version 8), having at least 1 measurable lesion according to the Response Evaluation Criteria in Solid Tumors (RECIST) $1.1,^{21}$ and the ability to receive oral treatment. Patient characteristics including age at diagnosis, sex, Eastern Cooperative Oncology Group performance status (PS) at the start of osimertinib treatment, smoking status, clinical stage, tumor histology, and brain metastasis status were identified via chart review. The Institutional Ethics Review Board of Kitasato University Hospital approved this prospective observational study, which was conducted in accordance with the tenets of the Declaration of Helsinki. All patients provided written informed consent before enrollment, after which they were treated with $80 \mathrm{mg}$ of osimertinib daily until disease progression or the development of unacceptable adverse events.

\section{Analysis of EGFR Mutations}

Initial testing for EGFR mutation status was performed using a sample of the primary tumor, a metastatic lesion, or pleural effusion fluid via the peptide nucleic acid-locked nucleic acid polymerase chain reaction clamp method or Oncomine Dx Target Test.

\section{Response Assessment}

After the initiation of osimertinib treatment, computed tomography of the chest and abdomen was performed every 2 to 3 months or at more frequent intervals if necessary. Positron emission tomography or bone scintigraphy as well as computed tomography or magnetic resonance imaging of the cranium were performed at 6-month intervals or whenever patients had significant symptoms associated with tumor lesions. The response to treatment was re-evaluated by two investigators (S.I. and M.S.).

\section{Toxicity Assessment and Dose Modification}

Toxicities were graded according to the National Cancer Institute Common Toxicity Criteria (version 5). Physical conditions, symptoms, blood tests, and chest X-rays of all patients were evaluated at an interval based on the discretion of the physician in charge throughout osimertinib treatment. Any interruption, discontinuation, or dose reduction of osimertinib caused by toxicities was at the discretion of the physician in charge.

\section{Statistical Analysis}

Progression-free survival (PFS) was defined as the interval between the date of osimertinib therapy initiation and that of disease progression or death; if neither occurred, the patient was censored on the date of the last follow-up examination. Time to treatment failure (TTF) was measured from the start of osimertinib therapy to either death, documentation of disease progression, or cessation of osimertinib therapy owing to the development of unacceptable toxicity. Survival curves were plotted using the Kaplan-Meier method, and differences according to PS status were 
analyzed using the Log rank test. Sex, smoking status, PS, stage, brain metastasis status, and EGFR genotype were used as variables analyzed via Cox proportional hazards models to predict the hazard ratios for PFS and TTF. A $P$-value $<0.05$ denoted statistical significance. All statistical analyses were performed using the SPSS software program, version 28.0 for Windows (IBM Corp., Armonk, NY, USA).

\section{Results}

\section{Patient Characteristics}

Forty-three patients with NSCLC who were treated with osimertinib between August 2018 and January 2021 were included in the final analysis. As shown in Table 1, the median patient age was 79 years; $65 \%$ were women and $77 \%$ had a good PS score (0 or 1). All patients had adenocarcinomas; $56 \%$ had an EGFR exon 19 deletion while $44 \%$ had an L858R point mutation. Moreover, $21 \%$ of the patients had brain metastasis.

\section{Response and Survival Analysis}

Table 2 shows the objective tumor responses. An objective response was obtained in 26 of the 43 patients, indicating an objective response rate (ORR) of $60.5 \%$ (95\% confidence interval [CI]: 44.9-76.0\%). A partial response was observed in 4 of the 10 patients with poor PS (ie, a score

Table I Patient Characteristics

\begin{tabular}{|l|c|}
\hline & $\mathbf{n = 4 3}(\%)$ \\
\hline $\begin{array}{l}\text { Age (years), median, range } \\
\text { Female/Male }\end{array}$ & $79(75-90)$ \\
\hline $\begin{array}{l}\text { Performance status } \\
0-1 / 2\end{array}$ & $24(56) / 19(44)$ \\
\hline $\begin{array}{l}\text { EGFR genotype } \\
\text { Del I9/L858R }\end{array}$ & $33(77) / 10(23)$ \\
\hline $\begin{array}{l}\text { Histology } \\
\text { Adenocarcinoma }\end{array}$ & $24(56) / 19(44)$ \\
\hline $\begin{array}{l}\text { Stage } \\
\text { IV/Recurrence }\end{array}$ & $43(100)$ \\
\hline $\begin{array}{l}\text { Smoking status } \\
\text { Current smoker } \\
\text { Never or former light smoker }\end{array}$ & $30(70) / 13(30)$ \\
\hline $\begin{array}{l}\text { Brain metastasis } \\
\text { Negative/Positive }\end{array}$ & 18 (42) \\
\hline
\end{tabular}

Table 2 Response to Osimertinib Therapy

\begin{tabular}{|l|c|}
\hline & $\mathbf{n = 4 3}$ \\
\hline Complete response & 0 \\
Partial response & 26 \\
Stable disease & 14 \\
Progressive disease & 3 \\
Response rate & $60.5 \%$ \\
$95 \% \mathrm{Cl}$ & $44.9-76.0$ \\
\hline
\end{tabular}

of 2), indicating a response rate of $40.0 \%$ (95\% CI: 19.0 $61.0 \%$ ); patients with favorable PS (scores of 0 or 1 ) had a higher response rate of $66.7 \%$ (95\% CI: $46.5-86.8 \%)$, although the difference was not significant $(P=0.17)$. Of the 9 patients pre-existing brain metastasis, an objective response was achieved in 4, indicating an ORR of $44.4 \%$. No disease progression owing to the exacerbation of brain metastatic lesions was observed among the 9 patients.

The cut-off date for survival analysis was July 2021, and the median follow-up period by that date was 17.2 months. The median PFS and TTF of the entire patient population were 22.1 months (95\% CI: $12.4-31.8$ months; Figure 1A) and 14.6 months (95\% CI: $12.3-16.9$ months; Figure 1B), respectively. The median PFS in the favorable and poor PS groups were 24.5 months (95\% CI: $11.3-37.7$ months) and 10.5 months (95\% CI: 3.1-17.9 months), respectively, with the difference just outside the threshold for significance $(P=0.068$; Figure $2 \mathrm{~A})$. The median TTF in the favorable and poor PS groups were 15.2 months (95\% CI: 5.3-25.1 months) and 5.8 months (95\% CI: 0.512.6 months), respectively; the difference was also just outside the threshold for significance $(P=0.063$; Figure 2B). The median PFS in patients without versus with brain metastasis was 24.5 months (95\% CI: 19.5-29.6 months) and 13.7 months (95\% CI: 6.1-23.1 months), respectively, although the difference was not significant $(P=0.14)$. The median TTF in patients without versus with brain metastasis were 15.0 months (95\% CI: 3.6-26.4 months) and 10.0 months (95\% CI: 0.5-22.3 months), respectively; the difference was not significant $(P=$ 0.17). The median PFS in patients with the exon 19 deletion versus those with the L858R mutation were 24.5 months (95\% CI: 9.7-39.2 months) and 22.1 months (95\% CI: 6.5-37.7 months), respectively, with no significant difference $(P=0.94)$. The median TTF in the exon 19 deletion versus L858R mutation groups were 13.7 months (95\% CI: 8.4-19.0 months) and 15.0 months (95\% CI: 
A

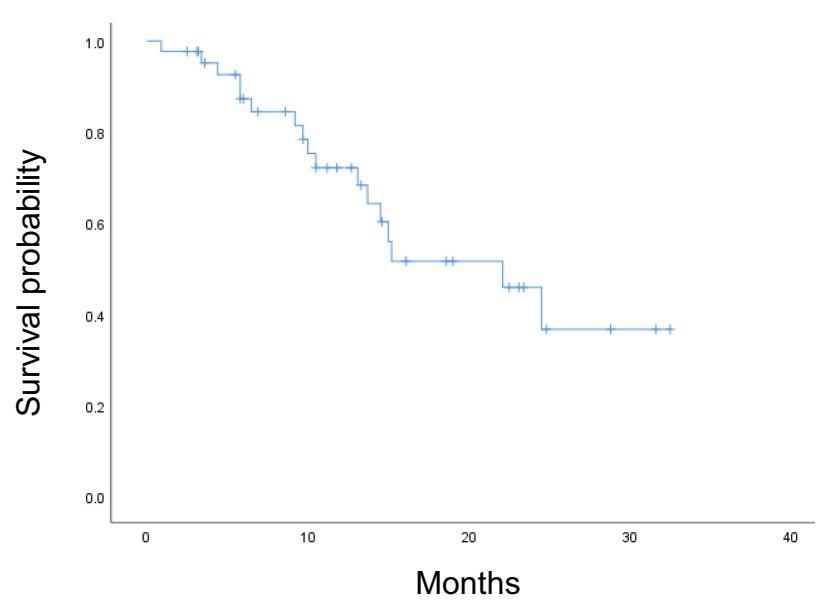

\begin{tabular}{ccc}
\hline $\mathrm{n}$ & PFS (months) & $95 \% \mathrm{Cl}$ \\
\hline 43 & 22.1 & $12.4-31.8$ \\
\hline
\end{tabular}

B

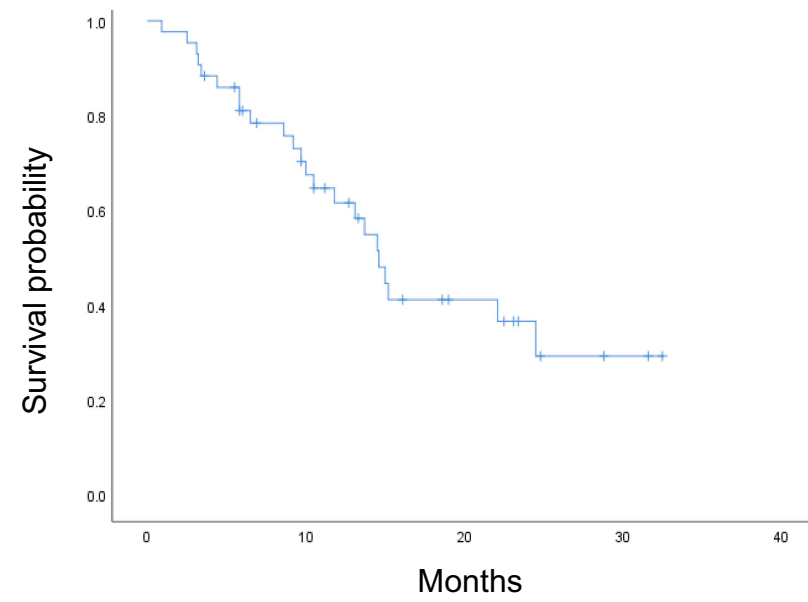

\begin{tabular}{ccc}
\hline $\mathrm{n}$ & TTF (months) & $95 \% \mathrm{Cl}$ \\
\hline 43 & 14.6 & $12.3-16.9$ \\
\hline
\end{tabular}

Figure I Kaplan-Meier curves showing (A) progression free survival (PFS) and (B) time to treatment failure (TTF) to osimertinib therapy.

A

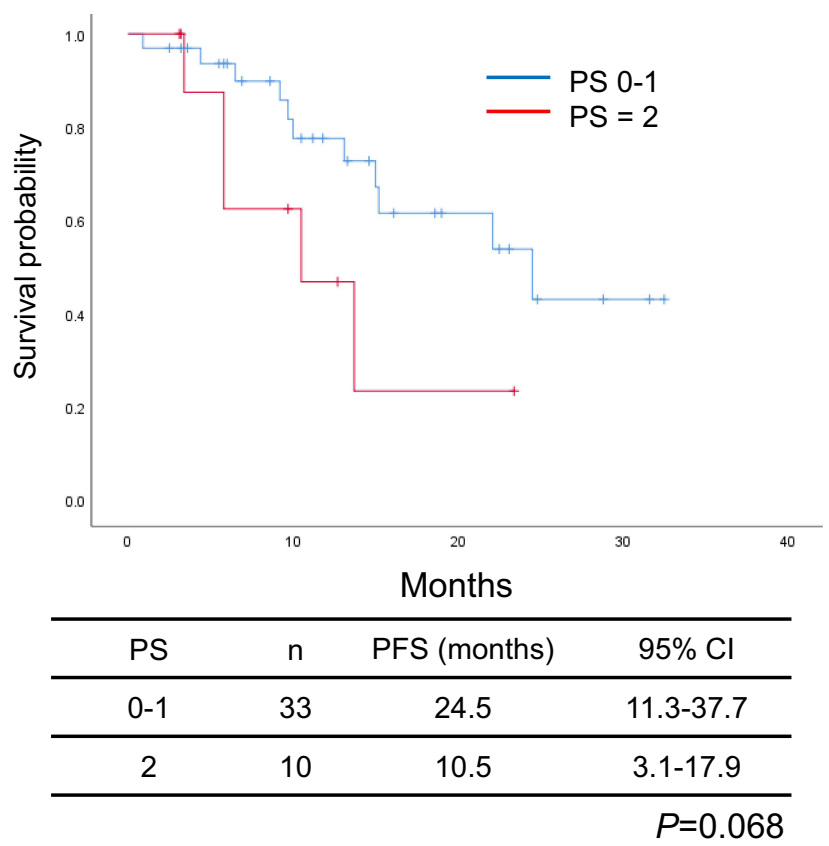

B

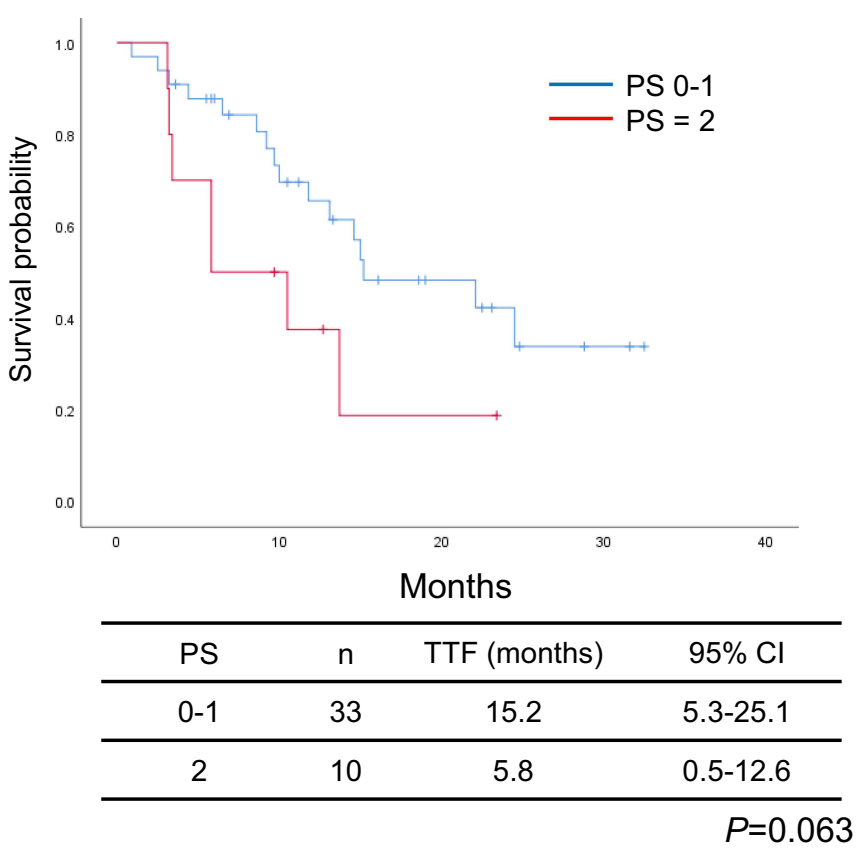

Figure 2 Kaplan-Meier curves showing (A) PFS and (B) TTF in patients receiving osimertinib therapy according to their Eastern Cooperative Oncology Group performance status score.

4.3-25.7 months), respectively, also with no significant difference $(P=0.70)$. Multivariate analysis identified PS and disease stage as independent predictors of PFS and TTF (Tables 3 and 4).

\section{Toxicities}

The toxicity of osimertinib was evaluated in all patients; the main adverse events observed during treatment are presented in Table 5. The most common was rash 
Table 3 Univariate and Multivariate Analyses for Factors That May Influence Progression-Free Survival

\begin{tabular}{|c|c|c|c|c|}
\hline \multirow[t]{2}{*}{ Variable } & \multicolumn{2}{|c|}{ Univariate Analysis } & \multicolumn{2}{|c|}{ Multivariate Analysis } \\
\hline & Hazard Ratio (95\% Cl) & $P$-value & Hazard Ratio (95\% Cl) & $P$-value \\
\hline \multicolumn{5}{|l|}{ Sex } \\
\hline Female & I (Ref.) & 0.68 & & \\
\hline Male & $1.22(0.47-3.19)$ & & & \\
\hline \multicolumn{5}{|c|}{ Performance status } \\
\hline $0-1$ & I (Ref.) & 0.08 & I (Ref.) & 0.021 \\
\hline 2 & $2.62(0.89-7.69)$ & & $3.94(1.23-12.7)$ & \\
\hline \multicolumn{5}{|l|}{ Smoking status } \\
\hline Never & I (Ref.) & 0.19 & Excluded & \\
\hline Smoker & $1.91(0.73-4.98)$ & & & \\
\hline \multicolumn{5}{|l|}{ Stage } \\
\hline Stage IV & I (Ref.) & 0.018 & I (Ref.) & 0.006 \\
\hline Recurrence & $0.31(0.12-0.82)$ & & $0.24(0.08-0.66)$ & \\
\hline \multicolumn{5}{|l|}{ Brain metastasis } \\
\hline Negative & I (Ref.) & 0.19 & Excluded & \\
\hline Positive & $2.00(0.70-5.7 I)$ & & & \\
\hline \multicolumn{5}{|c|}{ Type of EGFR mutation } \\
\hline L858R & I (Ref.) & & & \\
\hline Del. 19 & $0.91(0.35-2.40)$ & 0.86 & & \\
\hline
\end{tabular}

Abbreviations: $\mathrm{Cl}$, confidence interval; Del 19, exon 19 deletion; L858R, exon 21 point mutation.

Table 4 Univariate and Multivariate Analyses for Factors That May Influence Time to Treatment Failure

\begin{tabular}{|c|c|c|c|c|}
\hline \multirow[t]{2}{*}{ Variable } & \multicolumn{2}{|c|}{ Univariate Analysis } & \multicolumn{2}{|c|}{ Multivariate Analysis } \\
\hline & Hazard Ratio (95\% Cl) & $P$-value & Hazard Ratio (95\% Cl) & $P$-value \\
\hline \multicolumn{5}{|l|}{ Sex } \\
\hline Female & I (Ref.) & 0.65 & & \\
\hline Male & $1.21(0.53-2.75)$ & & & \\
\hline \multicolumn{5}{|c|}{ Performance status } \\
\hline $0-1$ & I (Ref.) & 0.047 & I (Ref.) & 0.015 \\
\hline 2 & $2.53(1.01-6.33)$ & & $3.35(1.27-8.86)$ & \\
\hline \multicolumn{5}{|l|}{ Smoking status } \\
\hline Never & I (Ref.) & 0.31 & & \\
\hline Smoker & $1.53(0.67-3.49)$ & & & \\
\hline \multicolumn{5}{|l|}{ Stage } \\
\hline Stage IV & I (Ref.) & 0.023 & I (Ref.) & 0.008 \\
\hline Recurrence & $0.38(0.17-0.88)$ & & $0.31(0.13-0.74)$ & \\
\hline \multicolumn{5}{|c|}{ Brain metastasis } \\
\hline Negative & I (Ref.) & 0.11 & Excluded & \\
\hline Positive & $2.05(0.84-4.99)$ & & & \\
\hline \multicolumn{5}{|c|}{ Type of EGFR mutation } \\
\hline L858R & I (Ref.) & & & \\
\hline Del. 19 & $0.91(0.40-2.08)$ & 0.87 & & \\
\hline
\end{tabular}


Table 5 Toxicities

\begin{tabular}{|c|c|c|c|c|c|}
\hline Adverse Event & Any Grade (\%) & Grade I (\%) & Grade 2 (\%) & Grade 3 (\%) & Grade 4 (\%) \\
\hline Rash acneiform & $18(42)$ & $13(30)$ & $5(12)$ & 0 & 0 \\
\hline Diarrhea & $14(33)$ & $14(33)$ & 0 & 0 & 0 \\
\hline Paronychia & $12(27)$ & $9(2 \mathrm{I})$ & $3(6)$ & 0 & \\
\hline Oral mucositis & $7(16)$ & $7(16)$ & 0 & 0 & 0 \\
\hline Anorexia & $6(13)$ & $5(\mathrm{II})$ & I (2) & 0 & 0 \\
\hline Dry skin & $3(6)$ & $3(6)$ & 0 & 0 & \\
\hline Fatigue & $3(6)$ & $2(4)$ & I (2) & 0 & \\
\hline Nausea & $2(4)$ & $2(4)$ & 0 & 0 & \\
\hline Dysgeusia & $2(4)$ & $2(4)$ & 0 & & \\
\hline Neutropenia & $7(16)$ & $4(10)$ & $2(4)$ & I (2) & 0 \\
\hline Leukopenia & $6(13)$ & $5(I I)$ & I (2) & 0 & 0 \\
\hline Anemia & $6(13)$ & $2(4)$ & I (2) & $3(7)$ & 0 \\
\hline Thrombocytopenia & $5(11)$ & $3(7)$ & $2(4)$ & 0 & 0 \\
\hline Creatinine increased & $5(11)$ & $3(7)$ & I (2) & I (2) & 0 \\
\hline AST/ALT increased & $4(10)$ & $4(10)$ & 0 & 0 & 0 \\
\hline QTc prolongation & $2(4)$ & 0 & $2(4)$ & 0 & 0 \\
\hline Interstitial lung disease & $8(18)$ & $4(10)$ & $3(6)$ & I (2) & 0 \\
\hline
\end{tabular}

acneiform (18 patients [42\%], none with grade $\geq 3$ ) followed by diarrhea (14 patients [33\%], none with grade $\geq 3$ ) and paronychia (12 patients [28\%], none with grade $\geq 3$ ). Interstitial lung disease (ILD) was detected in 8 patients (18.6\%), 1 of whom had grade 3 . The ILD occurred in the 8 patients were all manageable. Furthermore, 1 patient developed grade 3 neutropenia, while none developed febrile neutropenia. No treatment-related deaths occurred.

Thirteen patients $(30.2 \%)$ required osimertinib dose reduction owing to adverse events, while its administration was ceased in 6 patients $(14.0 \%)$ owing to ILD (from which they recovered after corticosteroid therapy). None of the patients discontinued osimertinib therapy because of other adverse events. Osimertinib administration was interrupted in 2 other patients with ILD until their conditions improved; subsequently, $40 \mathrm{mg}$ of osimertinib was administered per the physician's discretion. As a result, no recrudescence of drug-induced ILD was observed.

Twenty-two patients discontinued osimertinib treatment because of disease progression or ILD, among whom 11 received second-line treatments (other EGFRTKIs $[\mathrm{n}=5]$, platinum plus pemetrexed $[\mathrm{n}=5]$, and atezolizumab $[\mathrm{n}=1]$ ). Six patients with disease progression continued osimertinib despite progression; 3 of them were still receiving this agent by the data cut-off date. Twenty-one patients remained on osimertinib without disease progression. The osimertinib treatment durations until disease progression or cessation owing to adverse events in individual patients are summarized in Figure 3.

\section{Discussion}

The advent of targeted therapy has revolutionized treatments for a subset of patients with NSCLC, and testing newly diagnosed subjects for the presence of an EGFR mutation is now considered the standard of care. Our study found that first-line treatment with osimertinib provided a response rate of $60.5 \%$ and a median PFS of 22.1 months in elderly patients with NSCLC who harbor EGFR mutations. In the double-blind, phase III FLAURA study, osimertinib was found to significantly prolong PFS in comparison to gefitinib or erlotinib among patients with NSCLC who harbor sensitive EGFR mutations. ${ }^{8}$ Analysis of the Japanese subset of the FLAURA study revealed a PFS of 19.1 months in the osimertinib group, indicating the efficacy of first-line therapy with this agent in the Japanese population. ${ }^{22}$ Regarding osimertinib administration to the elderly population, the FLAURA study was notable in that it revealed the benefit of osimertinib over gefitinib and erlotinib with respect to PFS across different subgroups, including those categorized by age ( $<65$ vs $\geq 65$ years). ${ }^{8}$ However, while that study compared PFS according to the predefined subgroups of race (Asian vs non-Asian) and type of EGFR alteration (exon 19 deletion vs L858R mutation), age was not predefined for PFS analysis. Therefore, we concluded that none of the preexisting prospective studies had satisfactorily evaluated the efficacy and safety of osimertinib among elderly patients with NSCLC harboring sensitive EGFR mutations; to 


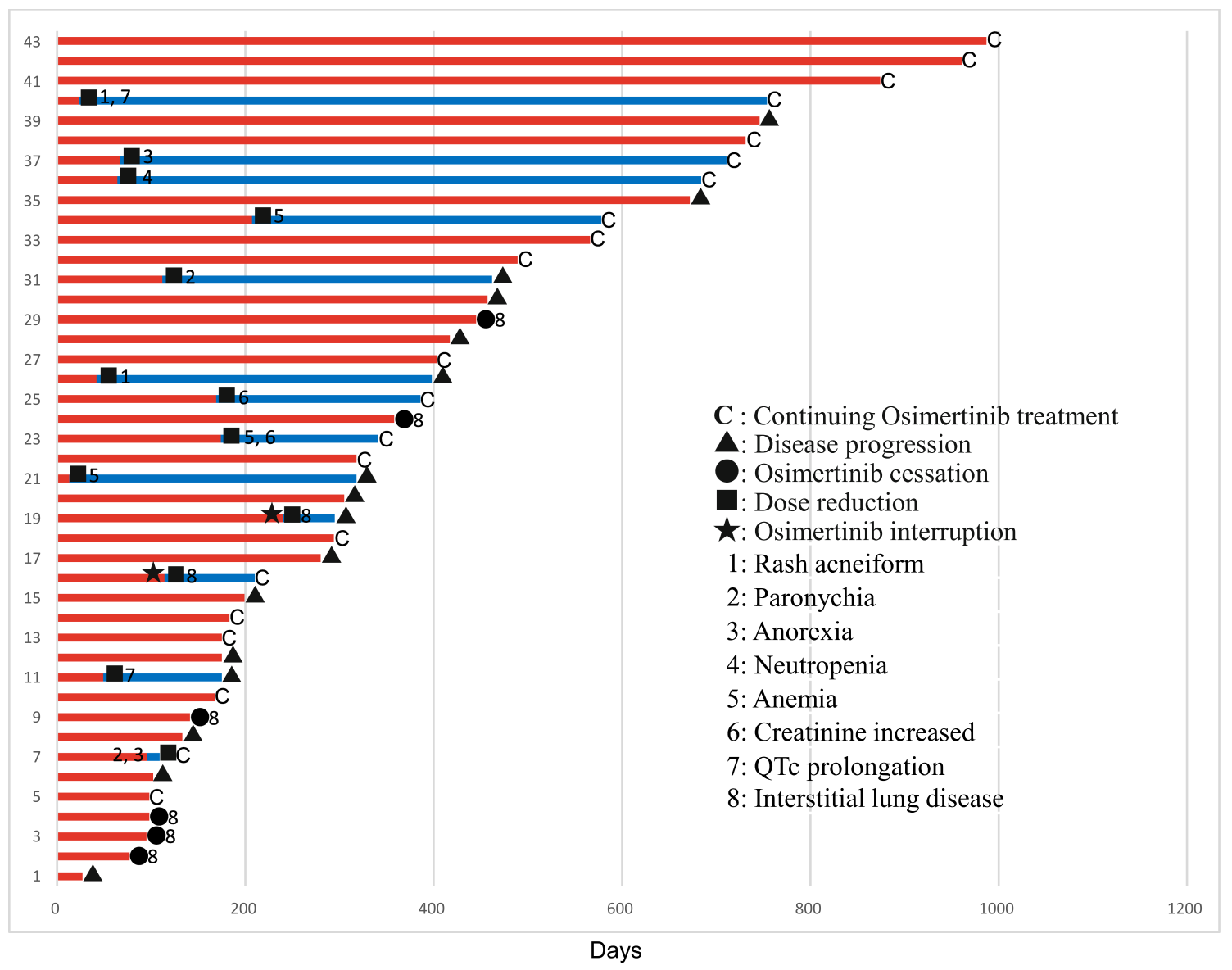

Figure 3 Durations of treatment with osimertinib before disease progression or cessation owing to adverse events in individual patients.

the best of our knowledge, ours is the first study to do so.

Notably, the PFS of 22.1 months owing to osimertinib therapy among the elderly population in our study was comparable to that derived from the analysis of the Japanese subset of FLAURA study. ${ }^{22}$ Previous studies have found that first-line EGFR-TKIs such as gefitinib, erlotinib, and afatinib are feasible and effective in elderly patients with NSCLC harboring EGFR mutations. ${ }^{11-20}$ Table 6 provides a summary of 4 prospective and 2 retrospective studies on patients $\geq 75$ years of age who were treated with EGFR-TKIs. The response rate in our present study was identical to that found in previous studies of first-line EGFR-TKI treatment in elderly patients with NSCLC harboring sensitive EGFR mutations; however, our patients' PFS appeared to be longer than that of patients who were treated with other EGFR-TKIs.

The PFS and TTF in our study were 22.1 and 14.6 months, respectively, indicating a lack of congruence between these parameters; this was attributed to treatment failure in the 6 patients who discontinued osimertinib owing to drug-induced ILD. Meanwhile, the 14.6-month TTF observed in our study was comparable to the PFS observed in previously reported elderly patients aged $\geq 75$ years who were administered other EGFR-TKIs, demonstrating the efficacy of osimertinib in this population. With respect to drug-induced ILD, its incidence in the Japanese subgroup of the FLAURA study was approximately $1.8 \%$ in those administered gefitinib but was $12.3 \%$ in those administered osimertinib. ${ }^{22}$ The findings of both our study and the FLAURA trial ${ }^{22}$ indicate that the incidence of osimertinib-induced ILD may be high among Japanese patients. Meanwhile, a previous study demonstrated that $80 \%$ of patients with ILD complicated by osimertinib recovered, and that mortality from druginduced ILD was lower in those administered osimertinib $(11.8 \%)$ than in those treated with gefitinib (38.9\%) and erlotinib $(35.6 \%) ;^{23}$ these data suggested that recovery from ILD can be expected in many patients receiving osimertinib. 


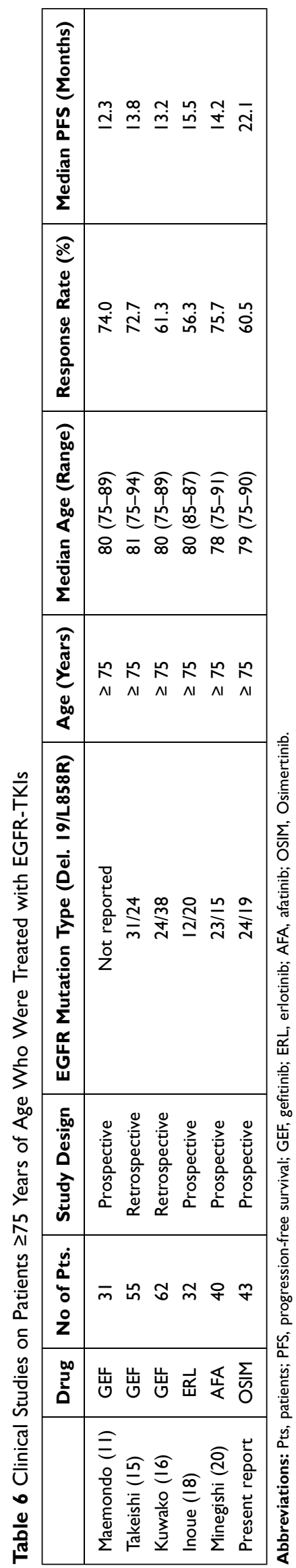

We identified poor PS as an independent predictor of unfavorable PFS and TTF among patients undergoing osimertinib therapy. Additionally, the response rate tended to be lower in patients with poor PS than in those with favorable PS, although the difference was not significant ( $40.0 \%$ vs $66.7 \%, P=0.17)$. Meanwhile, previous studies that comprised patients of all ages found that the PFS of those with poor PS who were treated with gefitinib and afatinib were 6.6 and 8.8 months, respectively. ${ }^{24,25}$ However, the PFS of patients with poor PS who were treated with osimertinib was significantly shorter than that of patients with favorable PS (10.5 vs 24.5 months); as such, the PFS of patients with poor PS treated with osimertinib appears to be comparable to that of counterparts administered other EGFR-TKIs. ${ }^{24,25}$

We also identified postoperative recurrence as a significant predictor of favorable PFS and TTF. Likewise, the phase III WJTOG3405 trial revealed that postoperative recurrence was independently associated with a longer PFS in patients receiving gefitinib. ${ }^{26}$ Moreover, we previously identified postoperative recurrence as a significant predictor of favorable PFS in patients with advanced NSCLC receiving the EGFRTKIs gefitinib and erlotinib. ${ }^{27}$ With respect to osimertinib, the present study is the first to prospectively identify postoperative recurrence as a significant favorable predictor of PFS. While the reasons for this remain unclear, we propose the following hypotheses. First, regular followup with imaging after curative surgery can detect early recurrence in the absence of symptoms; accordingly, the tumor burden of patients with recurrence is usually lower than that of those with stage IV NSCLC at diagnosis. Second, there is a higher possibility of tumor heterogeneity in patients with a large tumor burden; hence, anticancer drugs are less likely to be effective. ${ }^{28,29}$ The differences in tumor burden and heterogeneity between patients with stage IV disease and those who experience postoperative recurrence may therefore explain the more favorable PFS and overall survival in the latter group, particularly if they also harbor EGFR mutations. ${ }^{30-33}$

Regarding the type of EGFR mutations, no significant difference in either PFS or TTF was observed between patients with an exon 19 deletion and those with an L858R point mutation in the present study. The FLAURA study revealed a consistent PFS benefit for osimertinib treatment over first-generation EGFR-TKIs across all predefined subgroups that were assessed, including race (Asian vs non-Asian) and sensitive EGFR mutation type. ${ }^{8}$ 
Additionally, other previously conducted studies found no significant differences in PFS according to the type of sensitive EGFR mutation. ${ }^{34-36}$ This indicated that our findings with respect to the lack of PFS differences between patients with different $E G F R$ genotype were consistent with those of previous studies. As for brain metastases, the FLAURA study ${ }^{8}$ showed that central nervous system progression was less frequent in patients receiving osimertinib than in those receiving first-generation EGFRTKIs $(15 \%$ vs $6 \%)$. Other studies have also shown that osimertinib is effective against both systemic and brain metastatic lesions in patients with pre-existing brain metastases. ${ }^{37,38}$ In the present study, no statistically significant difference was observed in either PFS or TTF according to the presence or absence of brain metastases; as such, our findings were consistent with those of previous studies.

There were several limitations to our study. First, the sample size may not have been sufficiently large; moreover, the study was performed at a single institution. Second, although the individuals included in this study were elderly, data regarding their quality of life were not evaluated.

\section{Conclusion}

Osimertinib appears to be an effective and safe treatment option for elderly patients with advanced NSCLC harboring EGFR mutations. However, it is important to note the frequency of drug-induced ILD, which highlights the importance of a careful follow-up in this population. To obtain more conclusive results, additional studies in a larger elderly population are warranted.

\section{Research Involving Human Participants and/or Animals}

This study does not include human participants and animals.

\section{Data Sharing Statement}

The datasets generated and/or analyzed during the current study are available from the corresponding author upon reasonable request.

\section{Ethics Approval and Consent to Participate}

All procedures performed in studies involving human participants were in accordance with the ethical standards of the institutional and/or national research committee and with the 1964 Helsinki declaration and its later amendments or comparable ethical standards. This study was approved by the Institutional Ethics Review Board of Kitasato University Hospital. All patients gave informed consent for study participation.

\section{Informed Consent}

Informed consent was obtained from all individual participants included in the study.

\section{Consent for Publication}

All authors the study gave consent to publication of this study.

\section{Acknowledgments}

We gratefully thank the staff members of the Department of Respiratory Medicine, Kitasato University School of Medicine, for their suggestions and assistance.

\section{Author Contributions}

All authors contributed to data analysis, drafting or revising the article, have agreed on the journal to which the article will be submitted, gave final approval of the version to be published, and agree to be accountable for all aspects of the work.

\section{Funding}

There was no funding to declare.

\section{Disclosure}

Dr Takashi Sato reports personal fees from Chugai Pharmaceutical, Bristol Myers Squibb, Ono Pharmaceutical, and AstraZeneca, outside the submitted work. Dr Yoshiro Nakahara reports personal fees from BMS, Ono, Boehringer, AstraZeneca, Lilly, and Chugai; grants from Takeda and BMS, outside the submitted work. Prof. Dr. Jiichiro Sasaki reports personal fees from AstraZeneca, outside the submitted work. The authors report no other conflicts of interest in this work.

\section{References}

1. Maemondo M, Inoue A, Kobayashi K, et al. Gefitinib or chemotherapy for non-small-cell lung cancer with mutated EGFR. $N$ Engl J Med. 2010;362(25):2380-2388. doi:10.1056/NEJMoa0909530

2. Mitsudomi T, Morita S, Yatabe Y, et al. Gefitinib versus cisplatin plus docetaxel in patients with non-small-cell lung cancer harbouring mutations of the epidermal growth factor receptor (WJTOG3405): an open label, randomised Phase 3 trial. Lancet Oncol. 2010;11(2):121-128. doi:10.1016/S1470-2045(09)70364-X 
3. Rosell R, Carcereny E, Gervais R, et al. Erlotinib versus standard chemotherapy as first-line treatment for European patients with advanced EGFR mutation-positive non-small-cell lung cancer (EURTAC): a multicentre, open-label, randomised phase 3 trial. Lancet Oncol. 2012;13(3):239-246. doi:10.1016/S1470-2045(11)70393-X

4. Zhou C, Wu YL, Chen G, et al. Erlotinib versus chemotherapy as first-line treatment for patients with advanced EGFR mutation-positive non-small-cell lung cancer (OPTIMAL, CTONG-0802): a multicentre, open-label, randomised, phase 3 study. Lancet Oncol. 2011;12(8):735-742. doi:10.1016/S14702045(11)70184-X

5. Sequist LV, Yang JC, Yamamoto N, et al. Phase III study of Afatinib or cisplatin plus pemetrexed in patients with metastatic lung adenocarcinoma with EGFR mutations. J Clin Oncol. 2013;31 (27):3327-3334. doi:10.1200/JCO.2012.44.2806

6. Wu YL, Zhou C, Hu CP, et al. Afatinib versus cisplatin plus gemcitabine for first-line treatment of Asian patients with advanced non-small-cell lung cancer harbouring EGFR mutations (LUX-Lung 6): an open-label, randomised phase 3 trial. Lancet Oncol. 2014;15(2):213-222. doi:10.1016/S1470-2045(13)70604-1

7. Wu YL, Cheng Y, Zhou X, et al. Dacomitinib versus gefitinib as first-line treatment for patients with EGFR-mutation-positive non-small-cell lung cancer (ARCHER 1050): a randomised, open-label, phase 3 trial. Lancet Oncol. 2017;18(11):1454-1466. doi:10.1016/S1470-2045(17)30608-3

8. Soria JC, Ohe Y, Vansteenkiste J, et al. Osimertinib in untreated EGFR-mutated advanced non-small-cell lung cancer. $N$ Engl J Med. 2018;378(2):113-125. doi:10.1056/NEJMoa1713137

9. Barta JA, Zinner RG, Unger M. Lung cancer in the older patient. Clin Geriatr Med. 2017;33:563-577. doi:10.1016/j.cger.2017.06.008

10. Torre LA, Siegel RL, Ward EM, Jemal A. Global cancer incidence and mortality rates and trends - an update. Cancer Epidemiol Biomarkers Prev. 2016;25(1):16-27. doi:10.1158/1055-9965.EPI-15-0578

11. Maemondo M, Minegishi Y, Inoue A, et al. First-line gefitinib in patients aged 75 or older with advanced non-small cell lung cancer harboring epidermal growth factor receptor mutations: NEJ 003 study. J Thorac Oncol. 2012;7(9):1417-1422. doi:10.1097/JTO.0b0 $13 \mathrm{e} 318260 \mathrm{de} 8 \mathrm{~b}$

12. Fujita S, Katakami N, Masago K, et al. Customized chemotherapy based on epidermal growth factor receptor mutation status for elderly patients with advanced non-small-cell lung cancer: a phase II trial. BMC Cancer. 2012;12:185. doi:10.1186/1471-2407-12-185

13. Takahashi K, Saito H, Hasegawa Y, et al. First-line gefitinib therapy for elderly patients with non-small cell lung cancer harboring EGFR mutation: central Japan lung study group 0901. Cancer Chemother Pharmacol. 2014;74(4):721-727. doi:10.1007/ s00280-014-2548-z

14. Uruga H, Kishi K, Fujii T, et al. Efficacy of gefitinib for elderly patients with advanced non-small cell lung cancer harboring epidermal growth factor receptor gene mutations: a retrospective analysis. Intern Med. 2010;49(2):103-107. doi:10.2169/internalmedicine.49. 2531

15. Tateishi K, Ichiyama T, Hirai K, et al. Clinical outcomes in elderly patients administered gefitinib as first-line treatment in epidermal growth factor receptor-mutated non-small-cell lung cancer: retrospective analysis in a Nagano lung cancer research group study. Med Oncol. 2013;30(1):450. doi:10.1007/s12032-012-0450-2

16. Kuwako T, Imai H, Masuda $\mathrm{T}$, et al. First-line gefitinib treatment in elderly patients (aged $\geq 75$ years) with non-small cell lung cancer harboring EGFR mutations. Cancer Chemother Pharmacol. 2015;76 (4):761-769. doi:10.1007/s00280-015-2841-5

17. Morikawa N, Minegishi Y, Inoue A, et al. First-line gefitinib for elderly patients with advanced NSCLC harboring EGFR mutations. A combined analysis of North-East Japan study group studies. Expert Opin Pharmacother. 2015;16(4):465-472. doi:10.1517/14656566.20 15.1002396
18. Inoue $\mathrm{Y}$, Inui N, Asada $\mathrm{K}$, et al. Phase II study of erlotinib in elderly patients with non-small cell lung cancer harboring epidermal growth factor receptor mutations. Cancer Chemother Pharmacol. 2015;76 (1):155-161. doi:10.1007/s00280-015-2784-x

19. Imai H, Kaira K, Suzuki K, et al. A phase II study of Afatinib treatment for elderly patients with previously untreated advanced non-small-cell lung cancer harboring EGFR mutations. Lung Cancer. 2018;126:41-47. doi:10.1016/j.lungcan.2018.10.014

20. Minegishi Y, Yamaguchi O, Sugawara S, et al. A phase II study of first-line Afatinib for patients aged 75 years with EGFR mutation-positive advanced non-small cell lung cancer: North East Japan Study Group trial NEJ027. BMC Cancer. 2021;21(1):208. doi:10.1186/s12885-021-07861-1

21. Siegel R, DeSantis C, Virgo K, et al. Cancer treatment and survivorship statistics, 2012. CA Cancer J Clin. 2012;62(4):220-241. doi:10.3322/caac.21149

22. Ohe $\mathrm{Y}$, Imamura F, Nogami N, et al. Osimertinib versus standard-ofcare EGFR-TKI as first-line treatment for EGFRm advanced NSCLC: FLAURA Japanese subset. Jpn J Clin Oncol. 2019;49:29-36. doi:10.1093/jjco/hyy179

23. Gemma A, Kusumoto M, Sakai F, et al. Real-world evaluation of factors for interstitial lung disease incidence and radiologic characteristics in patients with EGFR T790M-positive NSCLC treated with Osimertinib in Japan. $J$ Thorac Oncol. 2020;15(12):1893-1906. doi:10.1016/j.jtho.2020.08.025

24. Okuma Y, Hosomi Y, Nagamata M, et al. Clinical outcomes after first-line EGFR inhibitor treatment for patients with NSCLC, EGFR mutation, and poor performance status. Anticancer Res. 2013;33 (11):5057-5064

25. Wu CE, Chang CF, Huang CY, et al. Feasibility and effectiveness of Afatinib for poor performance status patients with EGFR-mutationpositive non-small-cell lung cancer: a retrospective cohort study. BMC Cancer. 2021;21(1):859. doi:10.1186/s12885-021-08587-w

26. Yang JC, Wu Y, Schuler M, et al. Afatinib versus cisplatin-based chemotherapy for EGFR mutation-positive lung adenocarcinoma (LUX-Lung 3 and LUX-Lung 6): analysis of overall survival data from two randomised, phase 3 trials. Lancet Oncol. 2015;16 (2):141-151. doi:10.1016/S1470-2045(14)71173-8

27. Igawa $S$, Ryuge $S$, Ichinoe $M$, et al. Impact of EGFR-tyrosine kinase inhibitors on postoperative recurrent non-small-cell lung cancer harboring EGFR mutations. Oncol Res Treat. 2017;40(1-2):7-13. doi:10.1159/000455147

28. Park S, Holmes-Tisch AJ, Cho EY, et al. Discordance of molecular biomarkers associated with epidermal growth factor receptor pathway between primary tumors and lymph node metastasis in non-small cell lung cancer. $J$ Thorac Oncol. 2009;4(7):809-815. doi:10.1097/ JTO.0b013e3181a94af4

29. Monaco SE, Nikiforova MN, Cieply K, Teot LA, Khalbuss WE, Dacic S. A comparison of EGFR and KRAS status in primary lung carcinoma and matched metastases. Hum Pathol. 2010;41(1):94-102. doi:10.1016/j.humpath.2009.06.019

30. Taniguchi K, Okami J, Kodama K, Higashiyama M, Kato K. Intratumor heterogeneity of epidermal growth factor receptor mutations in lung cancer and its correlation to the response to gefitinib. Cancer Sci. 2008;99(5):929-935. doi:10.1111/j.1349-7006.2008.00782.x

31. Turner NC, Reis-Filho JS. Genetic heterogeneity and cancer drug resistance. Lancet Oncol. 2012;13(4):e178-e185. doi:10.1016/S14702045(11)70335-7

32. Park JH, Kim TM, Keam B, et al. Tumor burden is predictive of survival in patients with non-small-cell lung cancer and with activating epidermal growth factor receptor mutations who receive gefitinib. Clin Lung Cancer. 2013;14(4):383-389. doi:10.1016/j.cllc.2012.10.007

33. Liao S, Penney BC, Wroblewski K, et al. Prognostic value of metabolic tumor burden on 18F-FDG PET in nonsurgical patients with non-small cell lung cancer. Eur J Nucl Med Mol Imaging. 2012;39 (1):27-38. doi:10.1007/s00259-011-1934-6 
34. Yoshimura A, Yamada T, Okura N, et al. Clinical characteristics of osimertinib responder in non-small cell lung cancer patients with EGFR T790M mutation. Cancers. 2019;11(3):365. doi:10.3390/cancers1103 0365

35. Kuo CS, Huang C, Liu C, et al. Prior EGFR-TKI treatment in EGFR mutated NSCLC affects the allele frequency fraction of acquired T790M and the subsequent efficacy of Osimertinib. Target Oncol. 2019;14(4):433-440. doi:10.1007/s11523-019-00657-1

36. Su PL, Yang SC, Chen YL, et al. Real-world outcomes of NSCLC patients receiving tissue or circulating tumor DNA-guided osimertinib treatment. Cancer Med. 2019;8(13):5939-5947. doi:10.1002/cam4.2485
37. Mok TS, Wu YL, Ahn MJ, et al. Osimertinib or platinum-pemetrexed in EGFR T790M-positive lung cancer. $N$ Engl J Med. 2017;376(7):629-640. doi:10.1056/NEJMoa1612674

38. Yang JCH, Ahn MJ, Kim DW, et al. Osimertinib in pretreated T790M-positive advanced non-small-cell lung cancer: AURA study phase II extension component. J Clin Oncol. 2017;35(12):12 88-1296. doi:10.1200/JCO.2016.70.3223

\section{Publish your work in this journal}

Cancer Management and Research is an international, peer-reviewed open access journal focusing on cancer research and the optimal use of preventative and integrated treatment interventions to achieve improved outcomes, enhanced survival and quality of life for the cancer patient.
The manuscript management system is completely online and includes a very quick and fair peer-review system, which is all easy to use. Visit http://www.dovepress.com/testimonials.php to read real quotes from published authors. 